\title{
Lymphadenectomy after a positive sentinel node biopsy in patients with cutaneous melanoma. A systematic review
}

\author{
Maribel L. Da Cunha Cosme ${ }^{1 *} \mathbb{D}$, Juan F. Liuzzi Samaterra', Saul A. Siso Cardenas $^{1}$ and José I. Chaviano Hernández²
}

\begin{abstract}
Complete lymph node dissection (CLND) following a positive sentinel lymph node biopsy (SLNB) has been the standard treatment for years. However, there is increasing evidence that CLND could be omitted. Approximately $80 \%$ of patients with a positive sentinel node biopsy do not have additional nodal involvement; in these contexts, the SLNB could be diagnostic and therapeutic. However, in this group of patients, the therapeutic effect of CLND is unclear. A systematic search was performed in EMBASE and MEDLINE (PubMed), for studies published between January 1, 2014 and December 31, 2019. Studies were included when they compared immediate CLND and observation after a positive sentinel node. The outcomes of interest were: Overall Survival (OS), melanoma-specific survival (MSS), and disease-free survival (DFS).

Eleven studies met the inclusion criteria. Two randomized clinical trials reported no differences in OS or MSS when complete lymph dissection was compared with observation alone. An increase in regional relapse was observed in the CLND group, and in one randomized controlled trial $(R C T)$ the rate of disease-free survival was superior in those patients. Most populations in both RCTs had low sentinel lymph node biopsy (SLNB) metastatic deposits, and head and neck melanomas were not included or underrepresented. When CNLD was omitted, an active surveillance protocol was carried out.

The evidence supports that CLND in SLNB positive patients does not confer a survival benefit. Sentinel tumor burden, localization of primary tumor, and feasibility of active surveillance should be taken into account in treatment decisions.
\end{abstract}

Keywords: Melanoma, Lymphadenectomy, Positive sentinel node biopsy

\section{Introduction}

The incidence of cutaneous melanoma has been increasing over the recent decades and, currently, more than 130,000 cases occur globally each year (WHO, 2019). Surgery represents the mainstay of melanoma treatment; for regional disease, sentinel lymph node biopsy (SLNB) is recommended for patients with Breslow depth $>1 \mathrm{~mm}$ and also for patients with thinner melanomas and negative pathological features (Wong et al., 2018). Complete lymph node dissection (CLND), following a positive

\footnotetext{
* Correspondence: maribeldacunha@gmail.com

'Instituto Venezolano de los Seguros Sociales - Servicio Oncológico Hospitalario, Avenida Calvo Lairet, El Cementerio 1040. Distrito Capital, Caracas, Venezuela

Full list of author information is available at the end of the article
}

SLNB, has been the standard treatment for years. However, there is increasing evidence that CLND could be omitted.

The Multicenter Selective Lymphadenectomy Trial I (MSLT-I) demonstrated that sentinel node status is the most important prognostic factor for survival in patients with localized disease, and positive SLNB followed with CLND might improve the disease-free survival (Morton et al., 2014). Nevertheless, approximately $80 \%$ of patients with a positive sentinel node biopsy do not have additional nodal involvement, in these contexts the SLNB could be diagnostic and therapeutic. However, in this group of patients, the therapeutic effect of CLND is unclear (Morton et al., 2014).

(c) The Author(s). 2021 Open Access This article is licensed under a Creative Commons Attribution 4.0 International License, which permits use, sharing, adaptation, distribution and reproduction in any medium or format, as long as you give appropriate credit to the original author(s) and the source, provide a link to the Creative Commons licence, and indicate if changes were made. The images or other third party material in this article are included in the article's Creative Commons licence, unless indicated otherwise in a credit line to the material. If material is not included in the article's Creative Commons licence and your intended use is not permitted by statutory regulation or exceeds the permitted use, you will need to obtain permission directly from the copyright holder. To view a copy of this licence, visit http://creativecommons.org/licenses/by/4.0/. 
Surgical morbidity associated with CLND is variably reported in the literature, with overall complications ranging from 25 to 67\% (Wrightson et al., 2003; Theodore et al., 2017; Slagelse et al., 2018; Moody et al., 2017). Major complications occurred in $23-50 \%$ of patients after CLND, compared with 5-6\% after SLNB, and the quality of life was also worse in patients who underwent CLND (Wrightson et al., 2003; Moody et al., 2017; Egger et al., 2019; Gjorup et al., 1990).

Several retrospective trials have been carried out to elucidate which patients may benefit from CLND through an effort to avoid its associated morbidity. Recently, the final results of two large randomized controlled trials (RCTs) analyzing if CLND improves survival in positive SLNB have been published. These new studies could help to improve therapeutic decisions in the melanoma positive SLNB population (Faries et al., 2017; Leiter et al., 2019).

\section{Objectives}

To compare the benefits and harms of CLND in patients with cutaneous melanoma and positive SLNB.

\section{Methods}

\section{Sources / search strategy}

The literature search was done according to the Cochrane Guidelines for systematic reviews. A systematic search was performed through EMBASE and MEDLINE (PubMed), between January 1, 2014 and December 31, 2019. The search strategy combined keywords and MESH terms for "Lymph Node Excision", "Melanoma", and "Sentinel Lymph Node" (appendix 1). Additionally, a manual cross-reference search of eligible studies was done to find other relevant studies. Only English language studies were included. Each article title and abstract was reviewed by two authors and relevant publications were selected; differences were resolved by consensus. A summary of the search strategy is provided in Fig. 1.

\section{Types of studies and interventions}

We included all prospective and retrospective studies that compared immediate CLND and observation after a positive sentinel node.

\section{Types of participants}

Patients of any age and sex, with a diagnosis of cutaneous melanoma undergoing CLND after a positive SLNB.

\section{Types of outcome measures}

The outcomes of interest were:

Primary outcomes: Overall Survival (OS), melanomaspecific survival (MSS), and disease-free survival (DFS).
Secondary outcomes: the reported complication rates for SLNB and CLND. Principal exclusion criteria were lack of relevant outcome data.

\section{Data extraction, synthesis, and analysis}

A single data extraction was done, and it was verified by a second reviewer. The data presented in the selected studies were extracted and included in evidence tables. The abstracted data included: year of publication, study type, sample characteristics (age, gender, and location), Breslow depth, presence of ulceration, number of positive sentinel nodes, sentinel lymph node biopsy (SLNB) burden, median follow-up, OS, MSS, and DFS. Quality of evidence was assessed using GRADE methods. Table 1 summarizes the characteristics of the search of the studies included in this review.

\section{Results}

We screened 867 non-duplicate articles for eligibility and identified 22 potentially relevant studies for further review. Full-text screening excluded another 11 studies that did not fulfill the inclusion criteria. Finally, 11 studies were included. The total number of patients was 8664 , of those 6004 (69\%) underwent CLND, and 2660 (31\%) were observed. We identified two RCTs in which, following surgical excision of the primary cutaneous melanoma, CLND was compared with observation (Faries et al., 2017; Leiter et al., 2019), the remaining studies were observational and retrospective (Bamboat et al., 2014; Satzger et al., 2014; van der Ploeg et al., 2014; Melstrom et al., 2014; Gyorki et al., 2014; Fritsch et al., 2016; Mosquera et al., 2017; Lee et al., 2016; Klemen et al., 2019).

The Dermatological Cooperative Group - Selective Lymphadenectomy Trial (DeCOG-SLT), was a randomized clinical trial from 41 German Skin Cancer Centers; data was recollected from 2006 to 2014, and the final analysis (5 years follow up) was published in 2019. Positive SLNB patients were randomly assigned to undergo CLND or observation. Both groups were similar regard to age, gender, Breslow depth, ulceration, localization of the primary tumor, size of SLNB metastasis, and adjuvant therapy. Distant metastasis-free survival (DMFS) was analyzed as the primary endpoint, and DFS, OS, and recurrences in the regional lymph node basin were secondary endpoints. DeCOG-SLT was stopped prematurely due to recruiting problems, and the study finished under powered.

The Multicenter Selective Lymphadenectomy Trial II (MSLT-II) was a RCT whose data collection was from 2004 to 2014. Positive SLNB patients were randomly assigned to immediate CLND or nodal observation. There were no significant differences between groups about age, gender, primary tumor location, SLNB metastases burden, or adjuvant therapy. The primary outcome was a 3-year MSS. DFS, OS, nodal recurrence-free 
Records identified through database searching (EMBASE and PubMed) ( $n=1090)$
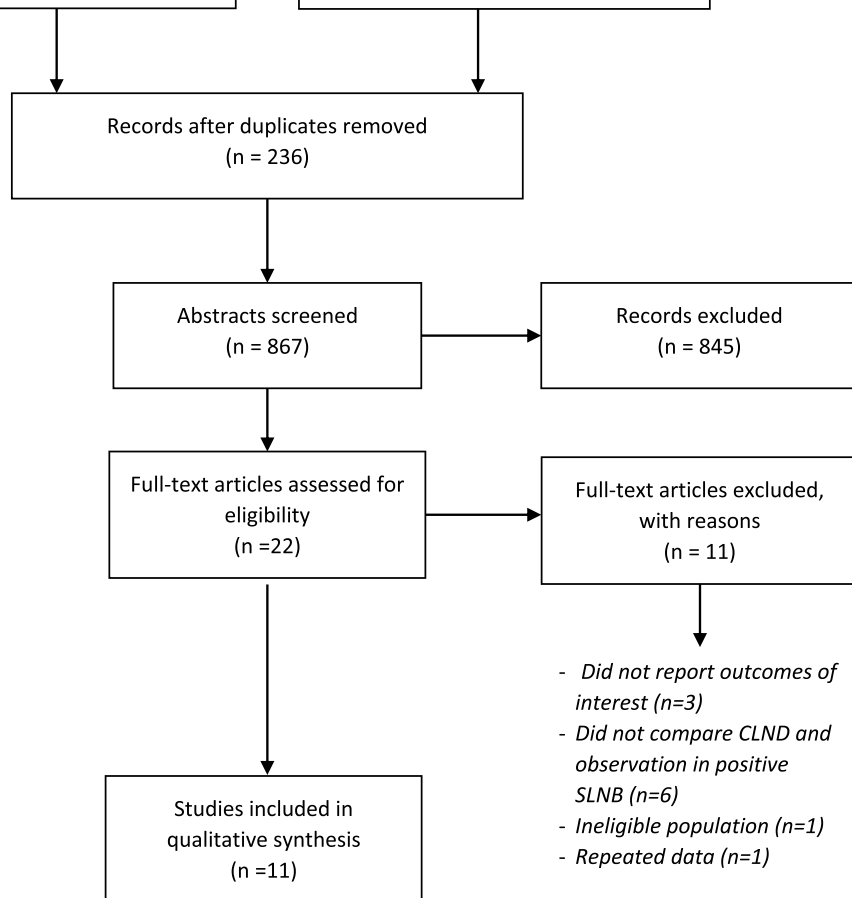

Fig. 1 PRISMA Flow Diagram

survival, the extent of nodal involvement, and DMFS were considered secondary outcomes.

In retrospective studies, the reasons for nodal observation were reported only twice, and the most commonly stated reason was "patient decision" (Bamboat et al., 2014; van der Ploeg et al., 2014). The median age of patients who underwent to immediate CLND was lower than in the observation group (Bamboat et al., 2014; Satzger et al., 2014; Melstrom et al., 2014; Gyorki et al., 2014; Fritsch et al., 2016; Lee et al., 2016) and ulceration was more frequent in CLND patients (Bamboat et al., 2014; Satzger et al., 2014; Melstrom et al., 2014; Gyorki et al., 2014; Fritsch et al., 2016; Mosquera et al., 2017; Klemen et al., 2019; Lee et al., 2004). The majority of patients were male. The presence of ulceration across studies ranged from 20 to 49\%, and DeCOG-SLT assumed that when not reported (32\%) it was negative.

Only one positive SLNB was reported in more than $70 \%$ of cases when retrospective studies were analyzed (Bamboat et al., 2014; Satzger et al., 2014; van der Ploeg et al., 2014; Mosquera et al., 2017; Lee et al., 2004). In regard to RCTs, MSLT-II reported one positive sentinel node in $79 \%$ of the cases and DeCOG-SLT in $92 \%$.
Positive SLNB patients are a heterogeneous group, and sentinel tumor burden is an important feature that should be recorded. The majority of retrospective studies did not report the sentinel tumor burden (Satzger et al., 2014; Melstrom et al., 2014; Gyorki et al., 2014; Fritsch et al., 2016; Mosquera et al., 2017; Klemen et al., 2019; Lee et al., 2004). In both RCTs, more than half of the patients had low volume disease $(\leq 1 \mathrm{~mm})$ at SLNB. This is an important fact when generalizing the results of these trials to other groups.

The sub-site of the primary cutaneous melanoma was defined in almost all studies. All retrospective data reported the limbs as the more frequent location. MSLT-II enrolled patients with cutaneous melanoma at any site $(86 \%$ trunk and extremities) while DeCOG-SLT did not include patients who had head and neck cutaneous melanoma.

Five retrospective studies did not describe surveillance protocols (Bamboat et al., 2014; Melstrom et al., 2014; Gyorki et al., 2014; Fritsch et al., 2016; Mosquera et al., 2017). When stated, observation always combined clinical examination with imaging (Satzger et al., 2014; van der Ploeg et al., 2014; Klemen et al., 2019; Lee et al., 2004). The MSLT-II and DeCOG-SLT patients were monitored 
Table 1 Characteristics of studies included in this review

\begin{tabular}{|c|c|c|c|c|c|c|}
\hline Author & Year & Study Method & Study Size & Obs. & CLND & $\begin{array}{l}\text { Quality of the evidence } \\
\text { (GRADE] }\end{array}$ \\
\hline Bamboat (Bamboat et al., 2014) & 2014 & Retrospective & 495 & 167 (34\%) & $328(66 \%)$ & Low \\
\hline Satzger (Satzger et al., 2014) & 2014 & Retrospective & 305 & $58(19 \%)$ & $247(81 \%)$ & Low \\
\hline Ploeg (van der Ploeg et al., 2014) & 2014 & Retrospective & 1539 & $158(10 \%)$ & $1381(90 \%)$ & Low \\
\hline Melstrom (Melstrom et al., 2014) & 2014 & Retrospective & 97 & $21(22 \%)$ & $76(78 \%)$ & Very low ${ }^{a}$ \\
\hline Gyorki (Gyorki et al., 2014) & 2014 & Retrospective & 54 & $18(33 \%)$ & $36(67 \%)$ & Very low ${ }^{b}$ \\
\hline Fritsch (Fritsch et al., 2016) & 2015 & Retrospective & 350 & $140(40 \%)$ & $210(60 \%)$ & Very low b,c \\
\hline Mosquera (Mosquera et al., 2017) & 2016 & Retrospective & 2172 & $716(33 \%)$ & $1456(67 \%)$ & Low \\
\hline Lee (Lee et al., 2016) & 2016 & Retrospective & 471 & $96(20 \%)$ & 375 (80\%) & Low \\
\hline Faries (Faries et al., 2017) & 2017 & $\mathrm{RCT}$ & 1755 & $931(53 \%)$ & $824(47 \%)$ & High \\
\hline Klemen (Klemen et al., 2019) & 2019 & Retrospective & 953 & $122(13 \%)$ & $831(87 \%)$ & Low \\
\hline Leiter (Leiter et al., 2019) & 2019 & $\mathrm{RCT}$ & 473 & 233 (49\%) & $240(51 \%)$ & High \\
\hline
\end{tabular}

Obs Observation, RCT Randomized Clinical Trial

GRADE Working Group grades of evidence

High quality: Further research is very unlikely to change our confidence in the estimate of effect

Moderate quality: Further research is likely to have an important impact on our confidence in the estimate of effect and may change the estimate

Low quality: Further research is very likely to have an important impact on our confidence in the estimate of effect and is likely to change the estimate

Very low quality: We are very uncertain about the estimate

${ }^{a}$ Downgraded for indirectness. Only evaluated patients with sentinel lymph nodes in more than one lymph node basin

${ }^{b}$ Downgraded for indirectness. Only evaluated patients with head and neck melanoma

${ }^{\mathrm{c}}$ Failure to identify the populations with CLND or SLNB only

by nodal ultrasound imaging and clinical examination every 4 months (MSLT-II) or every 3 months (DeCOGSLT) for the first 2 years, every 6 months for the next 3 years, and annually after 5 years. Additionally, the German study implemented serum S100b measurements every 3 months and whole-body CT scan, MRI, or PET-CT, or a chest $\mathrm{x}$-ray and abdomen sonography every 6 months.

Several outcomes were reported across studies. Three reported 5-year OS and none found statistically significant differences between patients treated with CNLS or observation (Leiter et al., 2019; Melstrom et al., 2014; Klemen et al., 2019). When 3 and 5-year MSS were evaluated, most studies also found no statistically significant difference (Faries et al., 2017; Bamboat et al., 2014; Satzger et al., 2014; Gyorki et al., 2014; Mosquera et al., 2017).

Melanoma specific survival, the MSLT-II primary endpoint, was essentially the same for both groups (86\% each, $P=0.42$ ) at 3 years, and no differences were found even after adjustment for other prognostic factors. However, at 3 years, the rate of DFS was slightly higher in the dissection group (68\% vs $63 \% ; P=0.05)$ and an increase in the rate of disease control in the regional nodes was observed in the CLND group (92\% vs $77, P<0.001$ ). Despite this, no significant differences in distant DMFS were detected between groups. As expected, more complications were found among patients who underwent CLND than observation. Lymphedema was more frequent in CLND than in the observation arm (24\% vs $6 \%$, $P<0.001 \%)$; when present, this condition was mostly mild $(64 \%)$ or moderate $(33 \%)$.
In DeCOG-SLT, both the 3 and 5-year DMFS rate were similar in CLND and observation arms, and statistically significant differences were not observed between them ( 3 years, $77 \%$ vs $74.9 \%, P=0.87$ and 5 years $67.6 \%$ vs $64.9 \%, P=0.87)$. No differences were evidenced either for DFS $(67.4 \%$ vs. $66.8 \%, P=0.75$ at 3 -years and $60.9 \%$ vs $59.9 \%, P=0.94$ at 5 -years $)$ or OS $(81.2 \%$ vs. $81.7 \%, P=$ 0.87 at 3 -years and $72.3 \%$ vs $71.4 \%, P=0.94$ at 5 -years).

DeCOG-SLT also evaluated adverse effects in the CLND arm and found that $24 \%$ developed adverse events; of these $13 \%$ were grade 3 or 4 toxicity. Lymphedema was the most frequent moderate-severe sideeffect reported. Lymph fistula, seromas, infection, and delayed wound healing were also described.

Tables 2 summarizes the characteristics of the patients included in the selected studies. Table 3 shows the outcomes comparing Observation vs CLND in SLN positive patients among the studies.

\section{Discussion}

Traditionally, for patients with a positive SLNB the standard of care was CLND, although recent evidence has supported the idea that SLNB alone could provide sufficient treatment for these patients. To elucidate that, we reviewed 11 studies (2 RCTs and 9 retrospective studies) that compared immediate CLND and observation after a positive sentinel node biopsy.

The majority of studies were retrospective, and patient selection bias in cohort studies reflected that most patients received CLND, especially if they were young and without 
comorbidities. Most studies included patients with primary melanoma of all sites. Two retrospective studies included only patients with head and neck melanoma, and these found no significant differences in 5-year MSS and RFS; however, both had a relatively short follow up period (Gyorki et al., 2014; Fritsch et al., 2016). Approximately 20 $28 \%$ of primary cutaneous melanomas arise in the head and neck (Wee et al., 2019; de Rosa et al., 2011), but this group was excluded from DeCOG-SLT and was under represented in MSLT-II. Since there is evidence that the location of head and neck melanoma is an independent adverse prognostic factor (Lachiewicz et al., 2008; Ettl et al., 2014; Tseng \& Martinez, 2011), the conclusions of these two trials should not be generalized to this specific group.

Some considerations should be taken into account about the benefits of CLND in melanoma patients. Positive SLNB represents the majority of patients who present with regional disease at diagnosis. It should be noted that this is a heterogeneous group, and 5-year survival rates range between 23 and 87\% (Balch et al., 2010). According to the American Committee on Cancer (AJCC) 8th edition, unless microsatellites, satellites or in-transit metastases are present, patients with clinically occult metastases (usually detected SLNB) are designated as N1a, N2a or N3a based on the number of tumor affected nodes, so the omission of CLND can downstage the final classification of the patient. Verver et al. reported an upstaging in N-category in 19\% of the patients and 5\% in AJCC stage based on the information offered by CLND.

Additional prognostic information obtained via CLND is not available in patients who are observed. In patients with positive non-sentinel nodes, the prognosis is similar to patients with palpable nodal disease (Balch et al., 2010; Leung et al., 2013; Rios-Cantu et al., 2017). It is expected that patients in the observation group have a higher risk of nodal recurrence. MSLT-II identified non-sentinel-node metastases in $11.5 \%$ of the patients in the dissection group, and these were a strong, independent prognostic factor for recurrence (hazard ratio, $1.78 ; P=0.005$ ); however, no significant difference in distant metastasis-free survival was detected between groups. DeCOG-SLT observed a slight improvement in the regional disease control rate in the CLND when compared with the observation group, though this was not significantly different.

Stratifying melanoma patients by primary tumor and SLNB findings could help in subsequent management recommendations. When a large EORTC multicenter cohort study evaluated this issue, they found that a stratification model incorporating ulceration and SLNB tumor burden showed at least similar performance compared with a model based on the CLND result (Balch et al., 2010).

There is evidence that tumor burden in SLNB is an important prognostic factor for survival, and is also predictive for additional non-sentinel positive nodes (van Akkooi et al., 2008; Cochran et al., 2004; Gershenwald et al., 2008; Ranieri et al., 2002; Satzger et al., 1990; Namikawa et al., 2019). The majority of patients included in MSLT-II and DeCOG-SLT had SLNB metastasis equal to or less than 1 $\mathrm{mm}$. Although subgroup analysis in both RCTs showed no differences in MSS or DMFS from CLND in patients with a larger sentinel tumor burden, the small number of patients could limit the statistical confidence of these results.

In addition to tumor burden, other genetic and immunological hallmarks have been studied to identify patients at high-risk of nodal relapse, however, to date, these have not been validated and are not currently used in clinical practice (Gerami et al., 2015; Berger et al., 2016; Farberg et al., 2017; Kakavand et al., 2015).

Based on results from MSLT-II and DeCOG-SLT demonstrating that CLND did not improve DSS or OS in patients with clinically occult nodal disease, the American Society of Clinical Oncology and Society of Surgical Oncology (ASCO/SSO) clinical practice guideline was updated, and the 2018 edition recommends that in the case of a positive SLNB, CLND or careful observation are valid options for patients with low-risk micro-metastatic disease (Wong et al., 2018). The European Society for Medical Oncology (ESMO) and Australian guidelines have also been updated and no longer recommend performing CLND dissection routinely when SLNB is positive (Dummer et al., 2015; Should all patients with a positive sentinel lymph node biopsy have a complete node dissection? Clinical Guidelines Wiki, 2020). All three studies emphasize that the risk of relapse, the possibility of frequent follow-up evaluations, and patient preferences should be taken into consideration in treatment choice.

Equivalent outcomes between observation and CLND groups are based on assuming that regular surveillance by high-quality ultrasound will identify recurrence earlier. In this context, both RCTs confirm that delayed CLND offers the same survival benefits as immediate CLND. When closing nodal observation is not feasible or if there is a concern for compliance, observation should not be recommended.

In MSLT-II, when compared with observation, CLND does not affect RFS, but improves regional control at 3 years. DeCOG has also reported that fewer regional lymph node recurrences occurred in the CLND group, although it was not statistically significant. However, more than $60 \%$ of patients in the DeCOG-SLT underwent treatment with adjuvant interferon, which may delay recurrence; moreover, follow up (median of 72.5 months) could have been too short for metastases in non-sentinel nodes become evident.

However, besides survival and risk of relapse, other factors must be taken into account in treatment decisions. Patients who underwent CLND showed a significantly higher rate of complications which can ultimately reduce the patient's quality of life. 


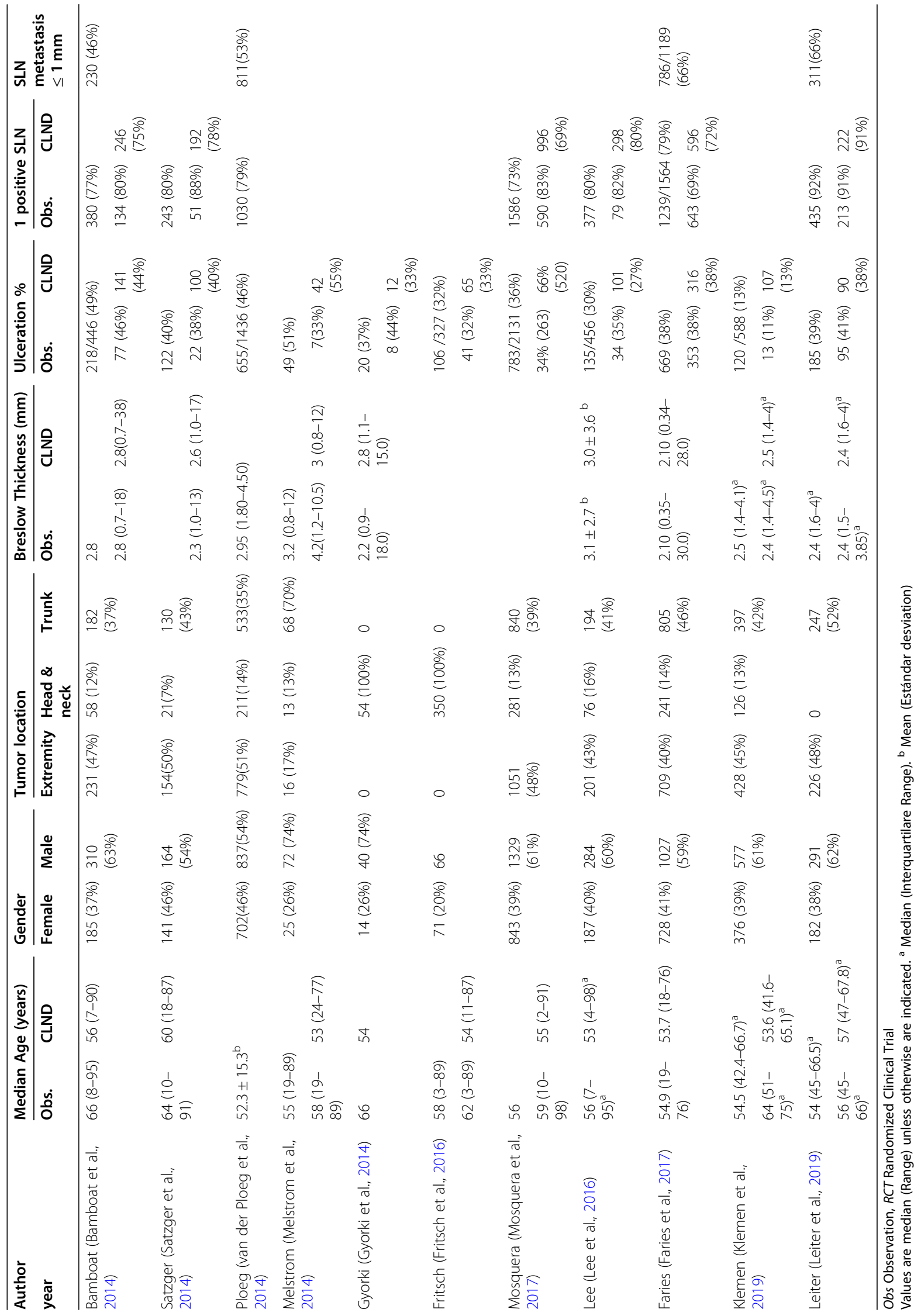


Table 3 Outcomes comparing Observation vs CLND in SLNB positive patients

\begin{tabular}{|c|c|c|c|c|c|c|c|c|c|c|c|c|c|c|c|c|c|c|}
\hline \multirow[t]{2}{*}{ AUTOR } & \multicolumn{2}{|c|}{ OS 3y } & \multirow{2}{*}{$p$} & \multicolumn{2}{|c|}{ OS 5y } & \multirow[t]{2}{*}{$p$} & \multicolumn{2}{|c|}{ MSS-3y } & \multirow[t]{2}{*}{$p$} & \multicolumn{2}{|c|}{ MSS-5y } & \multirow[t]{2}{*}{$p$} & \multicolumn{2}{|c|}{ DFS-3y } & \multirow[t]{2}{*}{$p$} & \multicolumn{2}{|c|}{ DFS-5y } & \multirow[t]{2}{*}{$p$} \\
\hline & Obs. & CLND & & Obs. & CLND & & Obs. & CLND & & Obs. & CLND & & Obs. & CLND & & Obs. & CLND & \\
\hline $\begin{array}{l}\text { Bamboat (Bamboat } \\
\text { et al., 2014) }\end{array}$ & & & & & & & $79 \%^{a}$ & $73 \%^{a}$ & 0.09 & $69 \%^{\mathrm{a}}$ & $62 \%^{a}$ & 0.09 & $37 \%^{a}$ & $50 \%^{a}$ & & $29 \%^{\mathrm{a}}$ & $41 \%^{a}$ & \\
\hline $\begin{array}{l}\text { Satzger (Satzger } \\
\text { et al., 2014) }\end{array}$ & & & & & & & & & & $81 \%^{\mathrm{a}}$ & $71 \%^{\mathrm{a}}$ & & & & & $71 \%^{a}$ & $56 \%^{\mathrm{a}}$ & \\
\hline \multicolumn{19}{|l|}{$\begin{array}{l}\text { Ploeg (van der Ploeg } \\
\text { et al., 2014) }\end{array}$} \\
\hline $\begin{array}{l}\text { Melstrom (Melstrom } \\
\text { et al., 2014) }\end{array}$ & & & & $35 \%^{a}$ & $37 \%^{a}$ & 0.6 & & & & & & & & & & & & \\
\hline $\begin{array}{l}\text { Gyorki (Gyorki } \\
\text { et al., 2014) }\end{array}$ & & & & & & & & & & & & & & & & $33 \%$ & $40 \%$ & 0.34 \\
\hline \multicolumn{19}{|l|}{$\begin{array}{l}\text { Fritsch (Fritsch } \\
\text { et al., 2016) }\end{array}$} \\
\hline $\begin{array}{l}\text { Mosquera (Mosquera } \\
\text { et al., 2017) }\end{array}$ & & & & & & & & & & $70 \%$ & $72 \%$ & 0.83 & & & & & & \\
\hline Lee (Lee et al., 2016) & & & & & & & & & & $66 \%$ & $74 \%$ & 0.015 & & & & $55 \%$ & $49 \%$ & 0.21 \\
\hline $\begin{array}{l}\text { Faries (Faries } \\
\text { et al., 2017) }\end{array}$ & & & & & & & $86 \%$ & $86 \%$ & 0.42 & & & & $63 \%$ & $68 \%$ & 0.05 & & & \\
\hline $\begin{array}{l}\text { Klemen (Klemen } \\
\text { et al., 2019) }\end{array}$ & & & & $59 \%$ & $64 \%$ & 0.084 & & & & $66 \%$ & $71 \%$ & 0.161 & & & & & & \\
\hline $\begin{array}{l}\text { Leiter (Leiter et al., } \\
\text { 2019) }\end{array}$ & $82 \%$ & $81 \%$ & 0.87 & $71 \%$ & $72 \%$ & 0.94 & & & & & & & $67 \%$ & $67 \%$ & 0.75 & $61 \%$ & $60 \%$ & 0.94 \\
\hline
\end{tabular}

Obs Observation, CLND Completion lymph node lymphadenectomy, Obs. Observation, OS Overall Survival, MSS Melanoma specific survival, DFS Disease free survival. ${ }^{a}$ Data extracted by software (PlotDigitizer $\left.{ }^{\oplus}\right)$

Finally, over recent years, important developments in adjuvant therapies have occurred, and new systemic therapies have been shown to improve DFS and OS in stage III melanoma patients (Kwak et al., 2019; Weber et al., 2017; Long et al., 2017; Eggermont et al., 2016; Eggermont et al., 2018; Maio et al., 2018). The number of patients diagnosed with stage III after wide local excision and a positive SLNB is growing. The presence of non-sentinel lymph node involvement at CLND is associated with increased mortality and DFS (Wiener et al., 2010; Ghaferi et al., 2009; Pasquali et al., 2014); this prognostic information will not be available in patients who underwent observation. Since the inclusion criteria for adjuvant trials in melanoma traditionally required primary tumor excision and CLND in case of positive SLNB, it is not clear if the recommendations for adjuvant treatment should be the same in the absence of CLND. Since the results of DeCOG and MSLT-II have already begun to influence clinical practice, tumor and SLNB characteristics should now be used to determine eligibility for adjuvant therapy.

New strategies must be developed to replace the prognostic information provided by the dissection since the decision to recommend adjuvant therapy after surgery should be based on the risk of recurrence or death, tumor features, risk of toxicities, and costs.

\section{Conclusions}

This systematic review shows there is strong evidence supporting that CLND in SLNB positive patients does not confer a survival benefit. These results, however, should be interpreted with caution, taking into account some facts. First, the majority of studies had SLNB deposits $\leq 1 \mathrm{~mm}$; thus, this group had a low risk of non-sentinel lymph node metastasis, so SLNB is more likely to be diagnostic and therapeutic. Second, head and neck melanomas were not included or were underrepresented, so in this circumstance the conclusions should not be extrapolated to this particular group. Finally, the observation is only comparable with CLND if the follow-up includes regular clinical examination and high-quality ultrasound. If this active surveillance protocol is not feasible, observation is not a safe option.

\footnotetext{
Abbreviations

CLND: Complete lymph node dissection; SLNB: Sentinel lymph node biopsy; OS: Overall Survival; MSS: Melanoma-specific survival; DFS: Disease-free survival; RCT: Randomized controlled trial; MSLT-I: Multicenter Selective Lymphadenectomy Trial I; DeCOG-SLT: Dermatological Cooperative Group Selective Lymphadenectomy Trial; DMFS: Distant metastasis-free survival; MSLT-II: Multicenter Selective Lymphadenectomy Trial II; AJCC: American Committee on Cancer; ASCO/SSO: American Society of Clinical Oncology and Society of Surgical Oncology; ESMO: European Society for Medical Oncology
}

Acknowledgements Not applicable. 


\section{Appendix}

Table 4 Search Strategy

\begin{tabular}{ll}
\hline Search engine & \\
\hline PubMed & Embase \\
\hline "Lymph Node Excision"[All Fields] & 'melanoma' AND 'sentinel lymph \\
AND "Melanoma"[All Fields] AND & node' AND 'lymph node \\
"Sentinel Lymph Node"[All Fields] & dissection' AND [2014-2019]/py \\
AND ("2014/01/01"[PDAT]: 2019/12/ & \\
31 "[PDAT]) &
\end{tabular}

\section{Authors' contributions}

Study conception and design: Maribel Da Cunha, Saul Siso, Juan Liuzzi. • Acquisition of data: Maribel Da Cunha, Saul Siso. • Analysis and interpretation of data: Maribel Da Cunha, José Chaviano, Juan Liuzzi. Drafting of manuscript: Maribel Da Cunha, José Chaviano. Critical revision of manuscript: Maribel Da Cunha, Juan Liuzzi, Saul Siso, José Chaviano.

\section{Funding}

Not applicable.

\section{Availability of data and materials}

Data sharing is not applicable.

\section{Ethics approval and consent to participate}

Not applicable.

\section{Consent for publication}

Not applicable.

\section{Competing interests}

The authors declare that they have no competing interests.

\section{Author details}

'Instituto Venezolano de los Seguros Sociales - Servicio Oncológico Hospitalario, Avenida Calvo Lairet, El Cementerio 1040. Distrito Capital, Caracas, Venezuela. ${ }^{2}$ Hospital Ana Francisca Perez de Leon, Avenida Francisco de Miranda, Sector El Llanito, 1020, Miranda, Caracas, Venezuela.

Received: 2 November 2020 Accepted: 11 December 2020

Published online: 12 January 2021

\section{References}

Balch CM, Gershenwald JE, Soong S-J et al (2010) Multivariate analysis of prognostic factors among 2,313 patients with stage III melanoma: comparison of nodal micrometastases versus macrometastases. J Clin Onco Off J Am Soc Clin Oncol 28:2452-2459. https://doi.org/10.1200/JCO.2009.27. 1627

Bamboat ZM, Konstantinidis IT, Kuk D et al (2014) Observation after a positive sentinel lymph node biopsy in patients with melanoma. Ann Surg Oncol 21: 3117-3123. https://doi.org/10.1245/s10434-014-3758-7

Berger AC, Davidson RS, Poitras JK et al (2016) Clinical impact of a 31-gene expression profile test for cutaneous melanoma in 156 prospectively and consecutively tested patients. Curr Med Res Opin 32:1599-1604. https://doi. org/10.1080/03007995.2016.1192997

Cochran AJ, Wen D-R, Huang R-R et al (2004) Prediction of metastatic melanoma in nonsentinel nodes and clinical outcome based on the primary melanoma and the sentinel node. Mod Pathol Off J U S Can Acad Pathol Inc 17:747755. https://doi.org/10.1038/modpathol.3800117

de Rosa N, Lyman GH, Silbermins D et al (2011) Sentinel node biopsy for head and neck Melanoma: A Systematic Review. Otolaryngol Neck Surg. https:// doi.org/10.1177/0194599811408554

Dummer R, Hauschild A, Lindenblatt N et al (2015) Cutaneous melanoma: ESMO clinical practice guidelines for diagnosis, treatment and follow-up. Ann Oncol Off J Eur Soc Med Oncol 26(Suppl 5):v126-v132. https://doi.org/10.1093/ annonc/mdv297

Egger ME, Scoggins CR, McMasters KM (2019) The sunbelt melanoma trial. Ann Surg Oncol. https://doi.org/10.1245/s10434-019-07828-4
Eggermont AMM, Blank CU, Mandala M et al (2018) Adjuvant Pembrolizumab versus placebo in resected stage III melanoma. N Engl J Med 378:1789-1801. https://doi.org/10.1056/NEJMoa1802357

Eggermont AMM, Chiarion-Sileni V, Grob J-J et al (2016) Prolonged survival in stage III melanoma with Ipilimumab adjuvant therapy. N Engl J Med 375: 1845-1855. https://doi.org/10.1056/NEJMoa1611299

Ettl T, Irga S, Müller S et al (2014) Value of anatomic site, histology and clinicopathological parameters for prediction of lymph node metastasis and overall survival in head and neck melanomas. J Cranio-Maxillo-fac Surg Off Publ Eur Assoc Cranio-Maxillo-fac Surg 42:e252-e258. https://doi.org/10.1016/ j.jcms.2013.09.007

Farberg AS, Glazer AM, White R, Rigel DS (2017) Impact of a 31-gene expression profiling test for cutaneous melanoma on dermatologists' clinical management decisions. J Drugs Dermatol JDD 16:428-431

Faries MB, Thompson JF, Cochran AJ et al (2017) Completion dissection or observation for sentinel-node metastasis in melanoma. N Engl J Med 376: 2211-2222. https://doi.org/10.1056/NEJMoa1613210

Fritsch VA, Cunningham JE, Lentsch EJ (2016) Completion lymph node dissection based on risk of nonsentinel metastasis in cutaneous melanoma of the head and neck. Otolaryngol--Head Neck Surg Off J Am Acad Otolaryngol-Head Neck Surg 154:94-103. https://doi.org/10.1177/0194599815605494

Gerami P, Cook RW, Wilkinson J et al (2015) Development of a prognostic genetic signature to predict the metastatic risk associated with cutaneous melanoma. Clin Cancer Res Off J Am Assoc Cancer Res 21:175-183. https:// doi.org/10.1158/1078-0432.CCR-13-3316

Gershenwald JE, Andtbacka RHI, Prieto VG et al (2008) Microscopic tumor burden in sentinel lymph nodes predicts synchronous nonsentinel lymph node involvement in patients with melanoma. J Clin Oncol Off J Am Soc Clin Oncol 26:4296-4303. https://doi.org/10.1200/JCO.2007.15.4179

Ghaferi AA, Wong SL, Johnson TM et al (2009) Prognostic significance of a positive nonsentinel lymph node in cutaneous melanoma. Ann Surg Oncol 16:2978-2984. https://doi.org/10.1245/s10434-009-0665-4

Gjorup CA, Groenvold M, Hendel HW et al (1990) (2017) health-related quality of life in melanoma patients: impact of melanoma-related limb lymphoedema. Eur J Cancer Oxf Engl 85:122-132. https://doi.org/10.1016/j.ejca.2017.07.052

Gyorki DE, Boyle JO, Ganly I et al (2014) Incidence and location of positive nonsentinel lymph nodes in head and neck melanoma. Eur J Surg Oncol J Eur Soc Surg Oncol Br Assoc Surg Oncol 40:305-310. https://doi.org/10.1016/ j.ejso.2013.11.017

Kakavand H, Vilain RE, Wilmott JS et al (2015) Tumor PD-L1 expression, immune cell correlates and PD-1+ lymphocytes in sentinel lymph node melanoma metastases. Mod Pathol Off J U S Can Acad Pathol Inc 28:1535-1544. https:// doi.org/10.1038/modpathol.2015.110

Klemen ND, Han G, Leong SP et al (2019) Completion lymphadenectomy for a positive sentinel node biopsy in melanoma patients is not associated with a survival benefit. J Surg Oncol 119:1053-1059. https://doi.org/10.1002/jso. 25444

Kwak M, Farrow N, Salama AK et al (2019) Updates in adjuvant systemic therapy for melanoma. J Surg Oncol 119:222-231. https://doi.org/10.1002/jso.25298

Lachiewicz AM, Berwick M, Wiggins CL, Thomas NE (2008) Survival differences between patients with scalp or neck melanoma and those with melanoma of other sites in the surveillance, epidemiology, and end results (SEER) program. Arch Dermatol 144:515-521. https:/doi.org/10.1001/archderm.144.4.515

Lee DY, Lau BJ, Huynh KT et al (2016) Impact of completion lymph node dissection on patients with positive sentinel lymph node biopsy in melanoma. J Am Coll Surg 223:9-18. https://doi.org/10.1016/j.jamcollsurg.2016.01.045

Lee JH, Essner R, Torisu-Itakura $\mathrm{H}$ et al (2004) Factors predictive of tumor-positive nonsentinel lymph nodes after tumor-positive sentinel lymph node dissection for melanoma. J Clin Oncol 22:3677-3684. https://doi.org/10.1200/ JCO.2004.01.012

Leiter U, Stadler R, Mauch C et al (2019) Final analysis of DeCOG-SLT trial: no survival benefit for complete lymph node dissection in patients with melanoma with positive sentinel node. J Clin Oncol 37:3000-3008. https:// doi.org/10.1200/JCO.18.02306

Leung AM, Morton DL, Ozao-Choy J et al (2013) Staging of regional lymph nodes in melanoma: a case for including nonsentinel lymph node positivity in the American joint committee on Cancer staging system. JAMA Surg 148:879. https://doi.org/10.1001/jamasurg.2013.3044

Long GV, Hauschild A, Santinami M et al (2017) Adjuvant Dabrafenib plus Trametinib in stage III BRAF-mutated melanoma. N Engl J Med 377:18131823. https://doi.org/10.1056/NEJMoa1708539 
Maio M, Lewis K, Demidov L et al (2018) Adjuvant vemurafenib in resected, BRAFV600 mutation-positive melanoma (BRIM8): a randomised, double-blind, placebo-controlled, multicentre, phase 3 trial. Lancet Oncol 19:510-520. https://doi.org/10.1016/S1470-2045(18)30106-2

Melstrom LG, Taylor E, Kuk D et al (2014) International multi-institutional management and outcome of melanoma patients with positive sentinel lymph nodes in more than one nodal basin. Ann Surg Oncol 21:4324-4329. https://doi.org/10.1245/s10434-014-3845-9

Moody JA, Botham SJ, Dahill KE et al (2017) Complications following completion lymphadenectomy versus therapeutic lymphadenectomy for melanoma - a systematic review of the literature. Eur J Surg Oncol J Eur Soc Surg Oncol Br Assoc Surg Oncol 43:1760-1767. https://doi.org/10.1016/j.ejso.2017.07.003

Morton DL, Thompson JF, Cochran AJ et al (2014) Final trial report of sentinelnode biopsy versus nodal observation in melanoma. N Engl J Med 370:599609. https://doi.org/10.1056/NEJMoa1310460

Mosquera C, Vora HS, Vohra N, Fitzgerald TL (2017) Population-based analysis of completion lymphadenectomy in intermediate-thickness melanoma. Ann Surg Oncol 24:127-134. https://doi.org/10.1245/s10434-016-5460-4

Namikawa K, Aung PP, Milton DR et al (2019) Correlation of tumor burden in sentinel lymph nodes with tumor burden in nonsentinel lymph nodes and survival in cutaneous melanoma. Clin Cancer Res Off J Am Assoc Cancer Res 25:7585-7593. https://doi.org/10.1158/1078-0432.CCR-19-1194

Pasquali S, Mocellin S, Mozzillo N et al (2014) Nonsentinel lymph node status in patients with cutaneous melanoma: results from a multi-institution prognostic study. J Clin Oncol 32:935-941. https://doi.org/10.1200/JCO.2013.50.7681

Ranieri JM, Wagner JD, Azuaje R et al (2002) Prognostic importance of lymph node tumor burden in melanoma patients staged by sentinel node biopsy. Ann Surg Oncol 9:975-981. https://doi.org/10.1007/bf02574515

Rios-Cantu A, Lu Y, Melendez-Elizondo V et al (2017) Is the non-sentinel lymph node compartment the next site for melanoma progression from the sentinel lymph node compartment in the regional nodal basin? Clin Exp Metastasis 34:345-350. https://doi.org/10.1007/s10585-017-9854-x

Satzger I, Leiter U, Gräger N et al (1990) (2019) melanoma-specific survival in patients with positive sentinel lymph nodes: relevance of sentinel tumor burden. Eur J Cancer Oxf Engl 123:83-91. https://doi.org/10.1016/j.ejca.2019.07.004

Satzger I, Meier A, Zapf A et al (2014) Is there a therapeutic benefit of complete lymph node dissection in melanoma patients with low tumor burden in the sentinel node? Melanoma Res 24:454-461. https://doi.org/10.1097/CMR. 0000000000000081

Should all patients with a positive sentinel lymph node biopsy have a complete node dissection? - Clinical Guidelines Wiki. https://wiki.cancer.org.au/australia/ Clinical_question:Should_all_patients_with_a_positive_sentinel_lymph_ node_biopsy_have_a_complete_node_dissection\%3F. Accessed 31 Jan 2020

Slagelse C, Munch T, Glazer C et al (2018) Natural history of pain associated with melanoma surgery. Pain Rep 3. https://doi.org/10.1097/PR9. 0000000000000689

Theodore JE, Frankel AJ, Thomas JM et al (2017) Assessment of morbidity following regional nodal dissection in the axilla and groin for metastatic melanoma. ANZ J Surg 87:44-48. https://doi.org/10.1111/ans.13526

Tseng WH, Martinez SR (2011) Tumor location predicts survival in cutaneous head and neck melanoma. J Surg Res 167:192-198. https://doi.org/10.1016/j. jss.2010.10.008

van Akkooi ACJ, Nowecki ZI, Voit C et al (2008) Sentinel node tumor burden according to the Rotterdam criteria is the most important prognostic factor for survival in melanoma patients: a multicenter study in 388 patients with positive sentinel nodes. Ann Surg 248:949-955. https://doi.org/10.1097/SLA. 0b013e31818fefe0

van der Ploeg APT, van Akkooi ACJ, Haydu LE et al (2014) The prognostic significance of sentinel node tumour burden in melanoma patients: an international, multicenter study of 1539 sentinel node-positive melanoma patients. Eur J Cancer 50:111-120. https://doi.org/10.1016/j.ejca.2013.08.023

Weber J, Mandala M, Del Vecchio M et al (2017) Adjuvant Nivolumab versus Ipilimumab in resected stage III or IV melanoma. N Engl J Med 377:18241835. https://doi.org/10.1056/NEJMoa1709030

Wee E, Wolfe R, Mclean C et al (2019) The anatomic distribution of cutaneous melanoma: a detailed study of 5141 lesions. Australas J Dermatol. https://doi. org/10.1111/ajd.13223

WHO | Skin cancers. In: WHO. http://www.who.int/uv/faq/skincancer/en/. Accessed 24 Nov 2019

Wiener M, Acland KM, Shaw HM et al (2010) Sentinel node positive melanoma patients: prediction and prognostic significance of nonsentinel node metastases and development of a survival tree model. Ann Surg Oncol 17: 1995-2005. https://doi.org/10.1245/s10434-010-1049-5

Wong SL, Faries MB, Kennedy EB et al (2018) Sentinel lymph node biopsy and Management of Regional Lymph Nodes in melanoma: American Society of Clinical Oncology and Society of Surgical Oncology clinical practice guideline update. J Clin Oncol 36:399-413. https://doi.org/10.1200/JCO.2017.75.7724

Wrightson WR, Wong SL, Edwards MJ et al (2003) Complications associated with sentinel lymph node biopsy for melanoma. Ann Surg Oncol 10:676-680. https://doi.org/10.1245/ASO.2003.10.001

\section{Publisher's Note}

Springer Nature remains neutral with regard to jurisdictional claims in published maps and institutional affiliations.
Ready to submit your research? Choose BMC and benefit from:

- fast, convenient online submission

- thorough peer review by experienced researchers in your field

- rapid publication on acceptance

- support for research data, including large and complex data types

- gold Open Access which fosters wider collaboration and increased citations

- maximum visibility for your research: over $100 \mathrm{M}$ website views per year

At BMC, research is always in progress.

Learn more biomedcentral.com/submissions 